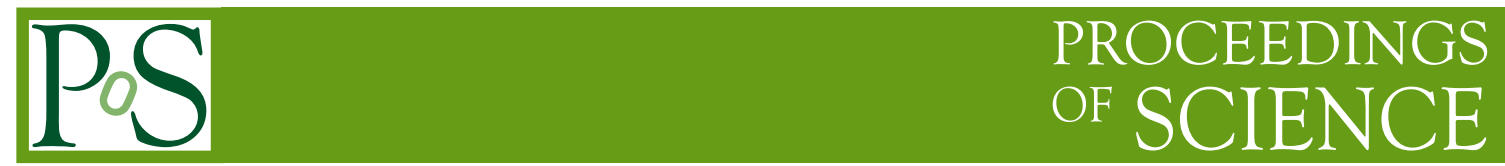

\title{
New Signatures of WIMPless Dark Matter
}

\author{
Jason Kumar* \\ University of Hawaii \\ E-mail: jkumar@hawaii.edu
}

\begin{abstract}
We consider new signatures of WIMPless dark matter, particularly those which can be used to test models with low dark matter mass. We focus on detection prospects at hadron colliders through the production of new heavy QCD-coupled particles, which decay to WIMPless dark matter plus jets. We find that the Tevatron can probe a significant fraction of the low mass parameter space with data already taken, and the LHC will have even better detection prospects with its first physics run.
\end{abstract}

Identification of Dark Matter 2010-IDM2010

July 26-30, 2010

Montpellier France

${ }^{*}$ Speaker. 


\section{Introduction}

There has been interest in low mass models of dark matter, especially since the DAMA collaboration reported $8.9 \sigma$ evidence of an annual modulation in their event rate [1], which could be consistent with dark matter with a low-mass $\left(m_{X} \sim \mathscr{O}(10) \mathrm{GeV}\right)$ and large spin-independent scattering cross-section $\left(\sigma_{\mathrm{SI}} \sim 10^{-2-5} \mathrm{pb}\right)$. This interest has been further heightened by recent data from other experiments. The CoGeNT experiment reported an unexplained event rate which is also consistent with a low-mass, large $\sigma_{\mathrm{SI}}$ dark matter model $[2,3]$. Finally, the CRESST collaboration has reported at this meeting a preliminary excess of events in the oxygen band, which could also be consistent with scattering of a low-mass dark matter particle [4]. A great question surrounding all of these signals is how they can be distinguished from an unknown background.

However, there have been contrary data from Xenon100 [5] and from CDMS [6], which are in tension with these low-mass signals. A new analysis of bounds from XENON10 and XENON100 [7] places constraints on low-mass dark matter as well. A new preliminary analysis of Xenon10 data, presented at this conference by Peter Sorenson [8], also exhibits significant tension with this data. However, even these analyses come with significant controversy [9].

The basic source of this controversy is the measurement of low recoil energies. Low mass dark matter tend to transfer less momentum to Standard Model nuclei during scattering, resulting in less recoil energy. For $m_{X} \lesssim 10 \mathrm{GeV}$, the recoil energies are near the threshold for many dark matter detectors. Essentially, the controversy surrounds whether or not experiments such as CDMS or XENON10/100 are truly sensitive to such low recoil energies.

It seems fair to say that the experimental status of low-mass dark matter is in a state of flux. In the face of this conflicting data one might be led to wonder, "What is a simple theorist to do?" In these proceedings, we will avoid the question of whether the experiments are actually seeing the interactions of a low-mass dark matter particle. Instead we will focus on two other questions:

- What theoretical models could yield a dark matter particle with low mass and large spinindependent scattering cross-section?

- What experiments could check this result, preferably with data which has either already been taken or will be taken soon?

To answer the first question, we will focus on WIMPless dark matter models [10]. Since the questions surrounding the experimental evidence arise from the problems which direct detection experiments often have in measuring low recoil energies, it is advisable to check these signals with methods very different from direct detection, and which do not face the difficulty of low recoil energy thresholds.

It was pointed out in $[11,12]$ that Super-Kamiokande is a very good experiment for checking these low-mass signals through searches for dark matter annihilating in the sun. Firstly, the neutrino flux due to dark matter annihilation is determined by solar dark matter capture rate, which is subject to fewer astrophysics uncertainties than other indirect detection search strategies. Secondly, SuperKamiokande is sensitive to very low-energy neutrinos, thus allowing it to probe the annihilation of low-mass dark matter. In particular, it was shown in [12] that Super-Kamiokande could probe this low-mass regime with the sample of fully-contained muons already taken in SK run III. 
In these proceedings, I will discuss another search strategy which is poised to probe low mass dark matter models with data already taken or soon to be available: collider experiments.

\section{WIMPless dark matter}

WIMPless dark matter is a hidden sector dark matter candidate which automatically has approximately the correct thermal relic density to explain our cosmological observations of dark matter, generalizing the "WIMP Miracle" beyond the case of weak interactions at the electroweak symmetry breaking scale [10]. In this model, both the hidden sector and MSSM sector receive SUSY-breaking through gauge-mediation from a single SUSY-breaking sector. The physics of the SUSY-breaking sector thus sets the soft breaking mass scale of the hidden sector and of the MSSM sector (which in turn sets the weak scale) through the relation

$$
\frac{g_{X}^{2}}{m_{X}}, \frac{g_{\text {weak }}^{2}}{m_{\text {weak }}} \propto \frac{M_{\text {mess }}}{F}
$$

where $g_{X}$ and $m_{X}$ are the coupling and soft SUSY-breaking scale of the hidden sector, $F$ is the SUSY-breaking $F$-term vev, and $M_{\text {mess. }}$ is the mass scale of the messengers. This relation is important because the thermal relic density is set by the annihilation cross-section, $\rho \propto\left\langle\sigma_{a n n} . v\right\rangle^{-1}$, which in turn is determined by the relation $\left\langle\sigma_{a n n .} v\right\rangle \propto \frac{g^{4}}{m^{2}}$. A stable particle at the hidden sector soft scale (the WIMPless candidate) then necessarily has approximately the same relic density as a WIMP.

The interesting feature of the model is that the relic density is very robust, and is independent of the details of the hidden sector. In particular, the model has approximately the correct density for a wide possible range of dark matter candidate mass, and regardless of whether the particle is a boson or fermion. This opens up the window for experimental search strategies beyond those typically used for WIMPs.

If the WIMPless particle is a scalar, then it can have a much larger $\sigma_{\mathrm{SI}}$ than one would expect from neutralino WIMPs. The basic reason is that scalar dark matter can couple to the Standard Model through a dimension 5 operator, $\left(X^{*} X\right)(\bar{f} f)$, while a Majorana fermion must couple through a dimension 6 operator, such as $(\bar{\chi} \chi)(\bar{f} f)$. This suggests that a scalar WIMPless candidate is a good candidate for matching these low mass, large $\sigma_{\mathrm{SI}}$ experimental signals.

WIMPless dark matter can couple to the SM sector through Yukawa couplings involving a 4th generation quark. In [13], a specific model was proposed to match the DAMA signal. In this model, dark matter couples exclusively to third generation quarks ${ }^{1}$ via the Yukawa coupling

$$
V=\lambda_{L} X \bar{Q}_{L\left(T^{\prime}, B^{\prime}\right)}^{\prime} q_{L(t, b)}+\lambda_{R t} X \bar{T}_{R}^{\prime} t_{R}+\lambda_{R b} X \bar{B}_{R}^{\prime} b_{R}+\text { h.c. }
$$

Gauge-invariance implies that the $Q_{L}^{\prime}=\left\{T_{L}^{\prime}, B_{L}^{\prime}\right\}, T_{R}^{\prime}, B_{R}^{\prime}$ form a mirror 4th generation multiplet. This multiplet must also be charged under the same hidden sector symmetry which stabilizes $X$, so it is really an exotic 4th generation quark multiplet. The mass of the 4th generation quarks is constrained by a combination of direct searches, perturbativity and precision electroweak constraints [14] to the the range $300 \mathrm{GeV} \lesssim m_{T^{\prime}, B^{\prime}} \lesssim 600 \mathrm{GeV}$.

\footnotetext{
${ }^{1}$ This is a simple way to satisfy flavor-changing neutral current constraints, while satisfying the observed Yukawa hierarchy, in which each generation mixes largely with the next generation.
} 
This WIMPless model couples to Standard Model nuclei through a loop of b quarks, which couples to the gluon content of the nucleus. To match these low mass signals with $300 \mathrm{GeV} \leq$ $m_{T^{\prime}, B^{\prime}} \leq 600 \mathrm{GeV}$ and $m_{X}=1-10 \mathrm{GeV}$, one would need $\lambda_{(L, R) b} \sim 0.3-1$ [13].

\section{Tests at hadron colliders}

Hadron colliders excel at the production of particles which couple to QCD. For this reason, search strategies at hadron colliders often focus on the production of strongly coupled particles (such as the gluino), which then decay to other particles (such as neutralinos). This strategy is especially important for dark matter, which is necessarily decoupled from QCD. Instead, hadron colliders produce heavy QCD-coupled particles which are also charged under a new symmetry, and then decay to the lightest particle charged under the same new symmetry, which is dark matter.

The simplest such decay we can write is $\phi_{\text {heavy }} \rightarrow \phi_{S M} X$, where $\phi_{\text {heavy }}$ is the the new heavy particle produced directly at the collider, $\phi_{S M}$ is a Standard Model particle which carries the same QCD quantum numbers as $\phi_{\text {heavy }}$, and $X$ is the QCD-neutral dark matter particle. Since the dark matter is necessarily long-lived and does not couple to strong or electromagnetic forces, this results in a collider signature of missing transverse energy. Collider experiments are thus ideally suited for testing low-mass dark matter models, as the event rate increases for smaller dark matter mass.

Supersymmetry analyses focus on the case where $\phi_{\text {heavy }}$ is either a color-octet fermion or a color-triplet scalar, and $m_{X} \sim 100-1000 \mathrm{GeV}$. WIMPless dark matter provides another simple example of this general set-up, where $\phi_{\text {heavy }}=T^{\prime}, B^{\prime}$ is a color-triplet fermion, which decays by $\phi_{\text {heavy }} \rightarrow b, t+X$. A general analysis of this possibility for both the Tevatron and LHC was performed in [15].

The process studied was the production of the up-type exotic quark through the process $p p(\bar{p}) \rightarrow$ $T^{\prime} \bar{T}^{\prime} \rightarrow X X t \bar{t}$. The signature searched for is missing energy, plus a large number of jets arising from top decay. Although the semi-leptonic decay channel was also studied, we found that the most promising channel was fully hadronic, since a lepton veto also rejects most Standard Model backgrounds with missing energy from neutrinos.

Initial cuts for the hadronic channel are

- No isolated electrons, muons or tau-tagged jets with $\left|p_{T}^{l}\right|>2 \mathrm{GeV}$.

- Minimum missing transverse energy: $E_{T}>100 \mathrm{GeV}$.

- At least 5 jets with $\left|p_{T}^{j}\right|>20 \mathrm{GeV}$ (Tevatron) or $\left|p_{T}^{j}\right|>40 \mathrm{GeV}$ (LHC).

- Minimum $\Delta \phi\left(\not p_{T}, p_{T}^{j}\right)$ for the leading jets: $\Delta \phi\left(\not p_{T}, p_{T}^{j 1}\right)>90^{\circ}$ and $\Delta \phi\left(\not p_{T}, p_{T}^{j 2}\right)>50^{\circ}$ (Tevatron); $\Delta \phi\left(\not p_{T}, p_{T}^{j}\right)>11.5^{\circ}$ for the first, second and third leading jets (LHC).

Additional cuts on $E_{T}$, the number of jets, and $H_{T}$ (defined by $H_{T}=\sum_{i=1}^{5}\left|p_{T}^{j}\right|$ ) are imposed point by point to maximize signal significance.

The main Standard Model backgrounds are $t \bar{t}$, leptonically-decaying $W+$ jets with a missed lepton or where a $\tau$ has been mistagged as a jet, and $Z+$ jets where $Z \rightarrow \bar{v} v$. The $\Delta \phi\left(\not p_{T}, p_{T}\right)$ cuts effectively eliminate the QCD multi-jet background. The signal and these backgrounds were simulated with Madgraph/MadEvent - Pythia 6.4.20 - PGS4 [16].

Detection prospects for the Tevatron and for the LHC at $10 \mathrm{TeV}$ center-of-mass energy are plotted in Figure 1. The detection prospects for $300 \mathrm{pb}^{-1}$ at the $\mathrm{LHC}$ at $10 \mathrm{TeV}$ roughly correspond 

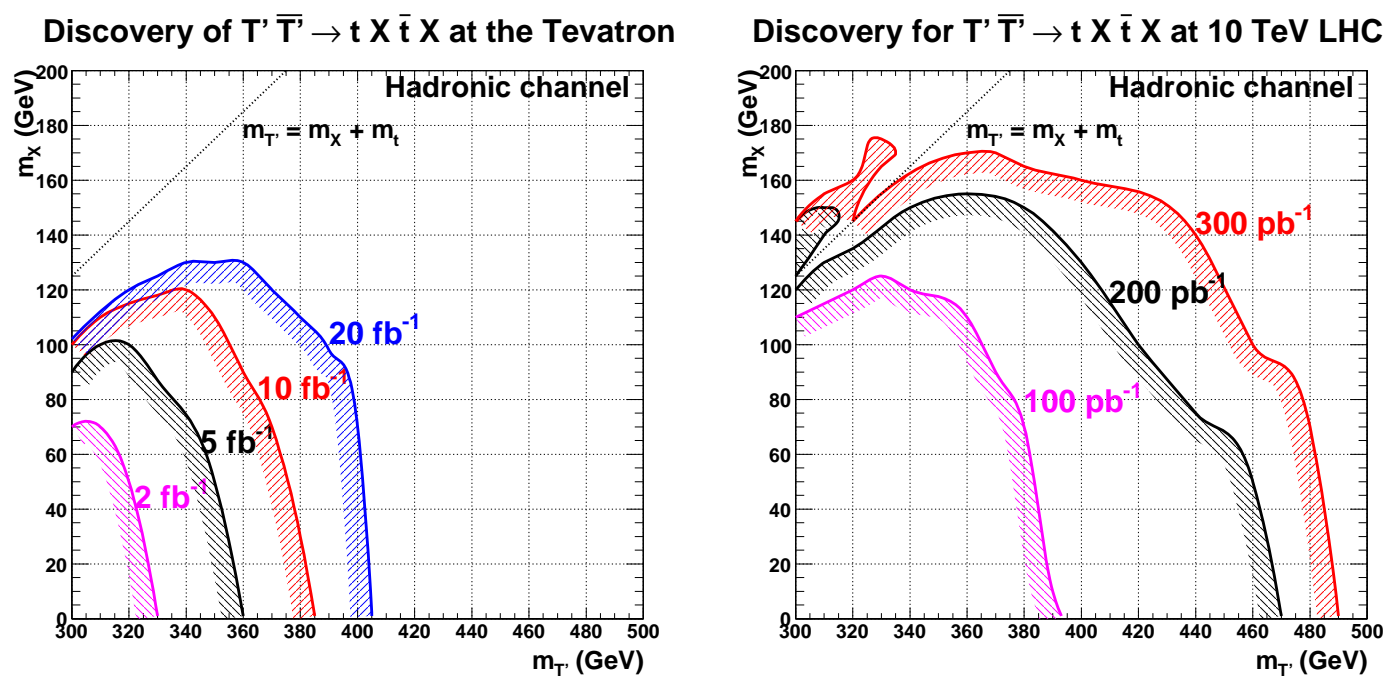

Figure 1: $3 \sigma$ (Gaussian equivalent) evidence contours for the hadronic channel for the Tevatron (left) with integrated luminosities 2, 5, 10, and $20 \mathrm{fb}^{-1}$ and for the LHC (right) with integrated luminosities 100, 200, and $300 \mathrm{pb}^{-1}$. For each point in parameter space, the cut with the best significance has been chosen.

to $1 \mathrm{fb}^{-1}$ for a $7 \mathrm{TeV}$ run. We thus see that the LHC can probe a significant fraction of the allowed parameter space in its first physics run.

\section{Conclusion}

We have found that hadron colliders can provide interesting new constraints on low mass models of dark matter which could match the data of DAMA, CoGeNT and CRESST, including WIMPless dark matter (see also [17]). The data and constraints in this region of parameter-space are constantly changing; indeed, during this conference we have seen a new preliminary analysis which can place tight constraints on this region of parameter space. As pointed out in [18], models which couple differently to protons and neutrons can potentially explain the low mass data while remaining consistent with even these new constraints. Since WIMPless models couple to the Standard Model via Yukawa couplings, it is easy to construct models whose couplings violate isospin (indeed, isospin violation should perhaps be expected). A study of such models is in preparation [19], and hadron colliders would again be an ideal venue for testing such models.

\section{Acknowledgments}

We are grateful to the organizers of IDM2010, and to J. Alwall, J. L. Feng, and S. Su for discussions and collaboration. JK is supported by DOE grant DE-FG02-04ER41291.

\section{References}

[1] R. Bernabei et al., arXiv:1002.1028 [astro-ph.GA].

[2] C. E. Aalseth et al. [CoGeNT collaboration], arXiv:1002.4703 [astro-ph.CO]. 
[3] D. Hooper, J. I. Collar, J. Hall and D. McKinsey, arXiv:1007.1005 [hep-ph].

[4] see talk of W. Seidel at IDM2010, http: //indico.in2p3. fr/contributionDisplay.py? contribId=195\&session Id=9\& confId=1565

[5] E. Aprile et al. [XENON100 Collaboration], arXiv:1005.0380 [astro-ph.CO].

[6] Z. Ahmed et al. [The CDMS-II Collaboration], arXiv:0912.3592 [astro-ph.CO]; D. S. Akerib et al. [CDMS Collaboration], arXiv:1010.4290 [astro-ph.CO].

[7] C. Savage, G. Gelmini, P. Gondolo and K. Freese, arXiv:1006.0972 [astro-ph.CO].

[8] See talk of P. Sorenson at IDM2010, http://indico.in2p3.fr/contributionDisplay.py? contribId=89\&session $I d=15$ \& conf $I d=1565$

[9] J. I. Collar and D. N. McKinsey, arXiv:1005.0838 [astro-ph.CO]; T. X. Collaboration, arXiv:1005.2615 [astro-ph.CO]; J. I. Collar and D. N. McKinsey, arXiv:1005.3723 [astro-ph.CO]; J. I. Collar, arXiv:1006.2031 [astro-ph.CO]; J. I. Collar, arXiv:1010.5187 [astro-ph.IM].

[10] J. L. Feng and J. Kumar, Phys. Rev. Lett. 101, 231301 (2008) [arXiv:0803.4196 [hep-ph]].

[11] D. Hooper, F. Petriello, K. M. Zurek and M. Kamionkowski, Phys. Rev. D 79, 015010 (2009) [arXiv:0808.2464 [hep-ph]].

[12] J. L. Feng, J. Kumar, J. Learned and L. E. Strigari, JCAP 0901, 032 (2009) [arXiv:0808.4151 [hep-ph]]; J. Kumar, J. G. Learned and S. Smith, Phys. Rev. D 80, 113002 (2009) [arXiv:0908.1768 [hep-ph]]; J. Kumar, arXiv:0903.1700 [hep-ph].

[13] J. L. Feng, J. Kumar and L. E. Strigari, Phys. Lett. B 670, 37 (2008) [arXiv:0806.3746 [hep-ph]].

[14] H. J. He, N. Polonsky and S. f. Su, Phys. Rev. D 64, 053004 (2001) [arXiv:hep-ph/0102144]; J. Alwall et al., Eur. Phys. J. C 49, 791 (2007) [arXiv:hep-ph/0607115]; G. D. Kribs, T. Plehn, M. Spannowsky and T. M. P. Tait, Phys. Rev. D 76, 075016 (2007) [arXiv:0706.3718 [hep-ph]]; R. Fok and G. D. Kribs, Phys. Rev. D 78, 075023 (2008) [arXiv:0803.4207 [hep-ph]]; B. Holdom, W. S. Hou, T. Hurth, M. L. Mangano, S. Sultansoy and G. Unel, PMC Phys. A 3, 4 (2009) [arXiv:0904.4698 [hep-ph]]; M. Hashimoto, arXiv:1001.4335 [hep-ph]; V. M. Abazov et al. [D0 Collaboration], Phys. Rev. Lett. 97, 171806 (2006) [arXiv:hep-ex/0608013]; T. Aaltonen et al. [CDF Collaboration], Phys. Rev. D 76, 072010 (2007) [arXiv:0707.2567 [hep-ex]]; T. Aaltonen et al. [CDF Collaboration], Phys. Rev. Lett. 102, 221801 (2009) [arXiv:0903.2618 [hep-ex]].

[15] J. Alwall, J. L. Feng, J. Kumar and S. Su, Phys. Rev. D 81, 114027 (2010) [arXiv:1002.3366 [hep-ph]].

[16] J. Alwall et al., JHEP 0709, 028 (2007) [arXiv:0706.2334 [hep-ph]]; T. Sjostrand, S. Mrenna and P. Skands, JHEP 0605, 026 (2006) [arXiv:hep-ph/0603175]; PGS - Pretty Good Simulator, http://www.physics.ucdavis.edu/ conway/research/software/ pgs/pgs4-general.html.

[17] J. Goodman, M. Ibe, A. Rajaraman, W. Shepherd, T. M. P. Tait and H. B. P. Yu, arXiv:1005.1286 [hep-ph]; arXiv:1008.1783 [hep-ph].

[18] S. Chang, J. Liu, A. Pierce, N. Weiner and I. Yavin, JCAP 1008, 018 (2010) [arXiv:1004.0697 [hep-ph]].

[19] J. L. Feng, J. Kumar, D. Marfatia and D. Sanford, in preparation. 